Proceedings of the 42th "Jaszowiec" International School and Conference on the Physics of Semiconductors, Wisła 2013

\title{
Temperature Dependences of Optical Energy Gaps of SbSI@CNT and SbSeI@CNT
}

\author{
M. JesioneK ${ }^{a, *}$, M. NOWAK ${ }^{a}$, M. KęPIŃSKA ${ }^{a}$ AND I. BEDNARCZYK ${ }^{b}$ \\ ${ }^{a}$ Solid State Physics Section, Institute of Physics, Silesian University of Technology \\ Z. Krasińskiego 8, 40-019 Katowice, Poland \\ ${ }^{b}$ Faculty of Materials Science, Silesian University of Technology, Z. Krasińskiego 8, 40-019 Katowice, Poland \\ This paper presents for the first time temperature dependences of optical energy gaps of SbSI@CNT and \\ SbSeI@CNT, i.e. carbon nanotubes (CNTs) filled with antimony sulfoiodide (SbSI) and antimony selenoiodide \\ (SbSeI). The heterostructures were prepared sonochemically using CNTs and elemental Sb, S or Se and I in the \\ presence of solvent under ultrasonic irradiation. Spectral characteristics of diffusive transmittance and reflectance \\ of SbSI@CNT and SbSeI@CNT were measured in temperature range $274 \mathrm{~K}<T<333 \mathrm{~K}$. The determinal \\ temperature dependence of indirect forbidden optical energy gap of SbSI@CNT has been fitted with $E_{\mathrm{gIf}}(T)=$ \\ $\left(1.92(2)-3.6(6) \times 10^{-4} \times T\right) \mathrm{eV}$. Indirect allowed optical energy gap of SbSeI@CNT has been fitted with $E_{\mathrm{gIa}}(T)=$ \\ $\left(1.817(5)-7.1(2) \times 10^{-4} \times T\right) \mathrm{eV}$.
}

DOI: $10.12693 /$ APhysPolA.124.836

PACS: 78.67.Ch, 62.23.Hj, 78.67.Uh, 81.07.Gf

\section{Introduction}

Carbon nanotubes (CNTs) have a lot of interesting properties for the physics and technology. Moreover, one knows that there are opportunities to influence on the properties of CNTs by filling them with various substances. An example of such substances can be the $\mathrm{A}^{\mathrm{V}} \mathrm{B}^{\mathrm{VI}} \mathrm{C}^{\mathrm{VII}}$-type materials, that are semiconducting ferroelectrics. Since photons in semiconductors generate excess free carriers, and induce a change of its electronic state, one may observe a lot of interesting phenomena in these materials.

Recently, the nanocrystalline, semiconducting ferroelectric antimony sulfoiodide (SbSI) and antimony selenoiodide (SbSeI) have been grown sonochemically in multiwalled CNTs $[1,2]$. The SbSI is a well known representative of the ternary chalcohalides that exhibits very attractive and suitable properties (see e.g., literature cited in [3]). The antimony selenoiodide belongs to the same group of materials. It should be underlined that $\mathrm{SbSeI}$ can be sonochemically prepared in the form of crystalline nanowires [4], too.

This work contains for the first time temperature dependences of optical energy gaps of SbSI@CNT and SbSeI@CNT, i.e. CNTs filled with SbSI and SbSeI.

\section{Experiment}

Details of the sonochemical preparation of SbSI@CNTs and SbSeI@CNTs were reported in $[1,2]$. The SbSI@CNTs and SbSeI@CNTs were characterized by using techniques such as powder X-ray diffraction (XRD), scanning electron microscopy (SEM), energy dispersive $\mathrm{X}$-ray analysis (EDS), high-resolution transmission elec-

*corresponding author; e-mail: Marcin.Jesionek@polsl.pl tron microscopy (HRTEM), and selected area electron diffraction (SAED).

Figure 1 presents line EDS analyses of chemical composition in direction perpendicular to two SbSeI@CNTs. Research have been carried out by microscope STEM HITACHI HD-2300A with linescan EDS. One can see the good feeling of CNTs with the SbSeI. The HRTEM and SAED investigations [2] proved the good crystallinity of the feeling. Similar results were obtained for SbSI in CNT [1].
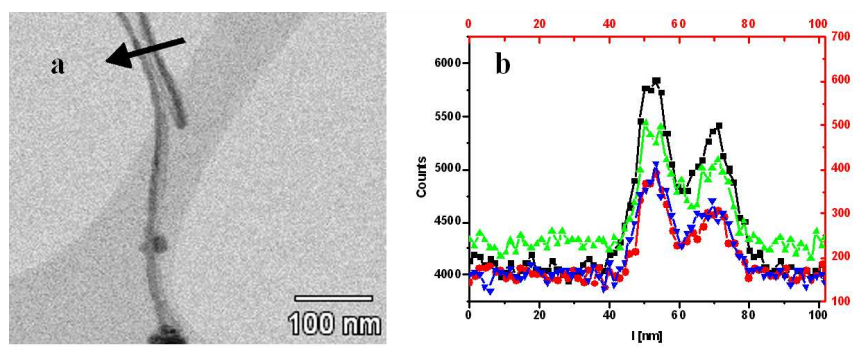

Fig. 1. (a) TEM image and (b) transversal distribution of elements in parallel SbSeI@CNT prepared in ethanol. Black arrow determines the line for which an EDS analysis of the chemical composition was made. Left scale

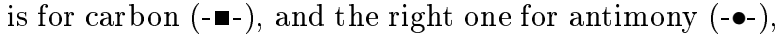
selenium (- $\left.\mathbf{\Delta}^{-}\right)$and iodine $(-\mathbf{\nabla}-)$.

Simultaneous measurements of optical diffuse reflection and transmission of thin films of SbSI@CNTs and SbSeI@CNTs deposited on BK-7 glass substrates were carried out inside vacuum chamber containing two integrating spheres (Ocean Optics Inc.). One of them measured the light diffusely transmitted through the sample while the second one (equipped with light source) determined the diffuse reflection. The chamber was also equipped with K2205 Cryogenic Microminiature Refrigeration II-B System and K-77 temperature controller 
(MMR Technologies, Inc.). Vacuum was obtained using TSH 071E turbomolecular drag pumping station (Pfeiffer). The optical signals were measured using PC2000 (Ocean Optics Inc.) spectrophotometer. The multiple averaged spectral characteristics containing 2048 data points for various wavelengths were registered using the OOI-Base program from Ocean Optics Inc. at constant temperature of the sample. The measurements were carried out in the temperature range from $274 \mathrm{~K}$ to $333 \mathrm{~K}$.

\section{Results and discussion}

According to the Kubelka-Munk theory diffusion reflectance $\left(R_{\mathrm{d}}\right)$ and diffusion transmission $\left(T_{\mathrm{d}}\right)$ for a plate of thickness $w$ are given by [5]:

$$
\begin{aligned}
& R_{\mathrm{d}}=\frac{\sinh \left(S_{\mathrm{KM}} y w\right)}{x \cosh \left(S_{\mathrm{KM}} y w\right)+y \sinh \left(S_{\mathrm{KM}} y w\right)}, \\
& T_{\mathrm{d}}=\frac{y}{x \cosh \left(S_{\mathrm{KM}} y w\right)+y \sinh \left(S_{\mathrm{KM}} y w\right)}, \\
& S_{\mathrm{KM}}=\frac{1}{y w} \ln \left(\frac{1-R_{\mathrm{d}}(x-y)}{T_{\mathrm{d}}}\right), \\
& A_{\mathrm{KM}}=(x-1) S_{\mathrm{KM}},
\end{aligned}
$$

where $S_{\mathrm{KM}}$ and $A_{\mathrm{KM}}$ are the Kubelka-Munk scattering and absorbance coefficients, respectively. Parameters $x$ and $y$ are calculated using the following formula:

$$
\begin{aligned}
& x=\frac{1+R_{\mathrm{d}}^{2}-T_{\mathrm{d}}}{2 R_{\mathrm{d}}}, \\
& y=+\sqrt{x^{2}-1} .
\end{aligned}
$$

The advantage of the Kubelka-Munk model is that the scattering and absorbance coefficients can be directly determined from the measured diffuse reflectance and diffuse transmission.

Knowing $R_{\mathrm{d}}(\lambda, T)$ and $T_{\mathrm{d}}(\lambda, T)$ and using Eqs. (3.1)(3.6), one can determine spectral characteristics of $A_{\mathrm{KM}}$ and $S_{\mathrm{KM}}$ for different temperatures.

Applying the method of simultaneous fitting of many mechanisms of absorption to the spectral dependence of $A_{\mathrm{KM}}$ [6], one can determine not only optical energy gap $\left(E_{\mathrm{g}}\right)$ but also the main mechanism of absorption in the investigated material.

For SbSI@CNT the best fitting was obtained for the sum of indirect forbidden absorption without excitons and phonon statistics $\left(E_{\text {gIf }}\right)$, free carrier absorption and constant absorption term. These results are consistent with those reported for SbSI@CNTs at room temperature in [1]. The best fitting for SbSeI@CNT was obtained for the sum of indirect allowed absorption without phonon statistics ( $\left.E_{\text {gIf }}\right)$ and constant absorption term $A_{0}$ associated with light scattering independent of the wavelength. There are the same mechanisms of light absorption as the reported for SbSeI@CNT at room temperature in [2].

Figure 2 shows the decrease of the values of the determined energy gaps of SbSI@CNT and SbSeI@CNT with increasing temperature. These energy gaps vary in ranges from $1.83 \mathrm{eV}(T=274 \mathrm{~K})$ to $1.80 \mathrm{eV}(T=$ $333 \mathrm{~K})$ and from $1.63 \mathrm{eV}(T=274 \mathrm{~K})$ to $1.58 \mathrm{eV}(T=$ $333 \mathrm{~K})$, respectively for SbSI@CNT and SbSeI@CNT.
For $274 \mathrm{~K}<T<333 \mathrm{~K}$ the evaluated indirect forbidden optical energy gap of SbSI@CNT has been fitted with $E_{\text {gIf }}(T)=\left(1.92(2)-3.6(6) \times 10^{-4} \times T\right) \mathrm{eV}$ (Fig. 2). In the same temperature range the evaluated indirect allowed optical energy gap of SbSeI@CNT has been fitted with $E_{\text {gIa }}(T)=\left(1.817(5)-7.1(2) \times 10^{-4} \times T\right) \mathrm{eV}$ (Fig. 2).

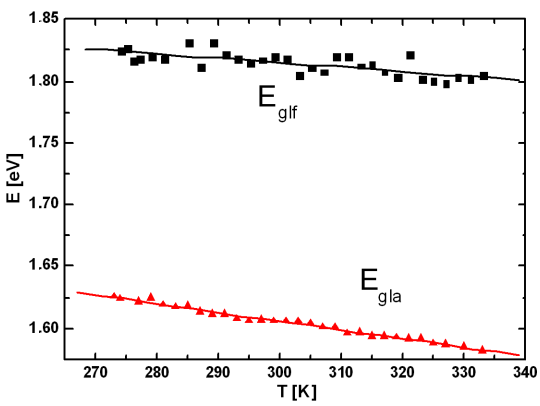

Fig. 2. Energy gap as a function of temperature for SbSI@CNT ( ) and SbSeI@CNT ( $₫$ ) prepared in methanol. Solid lines represent the least squares fitted temperature dependences (descriptions in the text).

Two facts are worth to underline: both materials have indirect band gaps, while they are forbidden and allowed, respectively for SbSI@CNT and SbSeI@CNT. This conclusion is consistent with the results published in the previous papers for SbSI nanowires [3] and SbSeI nanowires [4].

Obviously, the presented nanomaterials as the other one-dimensional semiconductor nanostructures should receive considerable attention from the scientific and engineering communities due to their potentially useful novel electronic properties. They have potentially great importance e.g. for the production of photovoltaic nanosensors.

\section{Acknowledgments}

This paper was partially supported by the National Science Centre Poland project no. 2011/01/B/ST5/ 06273.

\section{References}

[1] M. Nowak, M. Jesionek, P. Szperlich, J. Szala, T. Rzychoń, D. Stróż, Ultrason. Sonochem. 16, 800 (2009).

[2] M. Jesionek, M. Nowak, P. Szperlich, D. Stróż, J. Szala, K. Jesionek, T. Rzychoń, Ultrason. Sonochem. 19, 179 (2012).

[3] M. Nowak, P. Szperlich, Ł. Bober, J. Szala, G. Moskal, D. Stróż, Ultrason. Sonochem. 15, 709 (2008).

[4] M. Nowak, B. Kauch, P. Szperlich, M. Jesionek, M. Kepińska, Ł. Bober, J. Szala, G. Moskal, T. Rzychoń, D. Stróż, Ultrason. Sonochem. 16, 546 (2009).

[5] W.-F. Cheong, S.A. Prahl, A.J. Welch, IEEE J. Quantum. Electron. 26, 2166 (1990).

[6] M. Nowak, B. Kauch, P. Szperlich, Rev. Sci. Instrum. 80, 046107 (2009). 